\title{
Efectos colaterales positivos de la pandemia (II). Introducción a la e-Salud
}

\author{
Dr. Walter Reyes Caorsi
}

"Solamente el cambio es eterno, perpetuo, inmortal". Arthur Schopenhauer, filósofo alemán (1788-1860).

\section{Introducción y definiciones}

La pandemia ha permitido visualizar más claramente las prioridades de la humanidad, nunca tan globalizada como ahora, para las próximas décadas. Todo lo vinculado a la salud, que quizá hace unos meses no se percibía como prioridad, estará junto a los temas ambientales en los primeros lugares. Como hemos sido testigos, el coronavirus ha desnudado realidades en todo el mundo y también ha permitido visualizar oportunidades. En esta serie de artículos, que ambiciosamente hemos titulado Efectos colaterales positivos, intentamos jerarquizar algunas de esas oportunidades en el ámbito médico en general y en la cardiología en particular.

Una definición de telemedicina muy utilizada de la Organización Mundial de la Salud (OMS) jerarquiza el concepto de la distancia como un factor cla$\mathrm{ve}^{(1)}$ (tabla 1). Indudablemente se trata de un factor condicionante que de alguna manera obliga al uso de la telemedicina, pero su inclusión en la definición puede generar la impresión de que es una modalidad asistencial utilizable solo cuando existe esta limitación. Si asumiéramos una acepción más amplia del término "distancia”, podría enriquecerse el simple concepto geográfico. En este sentido, la OMS también establece: “...es el coste-efectivo de las tecnologías de la información y comunicación al servicio de la salud y sus campos relacionados, incluidos los servicios de salud, la vigilancia de la salud, la literatura en salud, la educación en salud, el conoci- miento y la investigación”. Queda, entonces, más claro que no se trata solo de un tema de distancia.

Cuando se menciona telemedicina es muy común que se identifique con la teleconsulta. Es una interpretación simplista que induce en forma inmediata a crear una falsa oposición entre lo presencial y lo virtual. Esta controversia no debería plantearse, pues a nuestro criterio no existe. La teleconsulta jamás podrá sustituir el vínculo presencial, personal y único, entre el médico y su paciente. Su objetivo, por el contrario, es mejorarlo, actualizarlo, facilitarlo y complementarlo, sobre todo desde el punto de vista del paciente, nunca sustituirlo. Tampoco es algo particularmente novedoso (figura 1). Todos, de diferentes maneras, practicamos telemedicina, aun sin ser conscientes de ello (teléfono, Whatsapp, Telegram, correo electrónico, aplicaciones de consulta o información bibliográfica, etcétera), y en general de manera desordenada. Resulta interesante recordar que en 1905, W. Einthoven, quien contribuyó de manera fundamental al desarrollo de la electrocardiografía, transmitió los registros obtenidos con su galvanómetro a través del teléfono a su hospital(2).

La teleconsulta constituye en definitiva un aspecto más de la llamada e-Salud, concepto mucho más complejo y abarcativo. En la era web se llamaba telemedicina, actualmente, en la era digital se llama e-Salud. Quizá en el futuro cercano empecemos a estar también familiarizados con la denominada m-Salud, o salud móvil, o la p-Salud, o salud personalizada, que serán herramientas básicas, en principio para el manejo del paciente crónico.

Recientemente, la OMS ha definido de forma más general y conceptual la e-Salud como "el uso de las tecnologías de la información y comunicación en apoyo de la salud y aspectos relacionados"(3). En la

Comisión Honoraria para la Salud Cardiovascular. Montevideo, Uruguay.

Correspondencia: Dr. Walter Reyes Caorsi. Correo electrónico: wreyes001@gmail.com

$\mathrm{El}$ autor declara no tener conflictos de intereses.

Recibido Ago 26, 2020; aceptado Set 2, 2020 


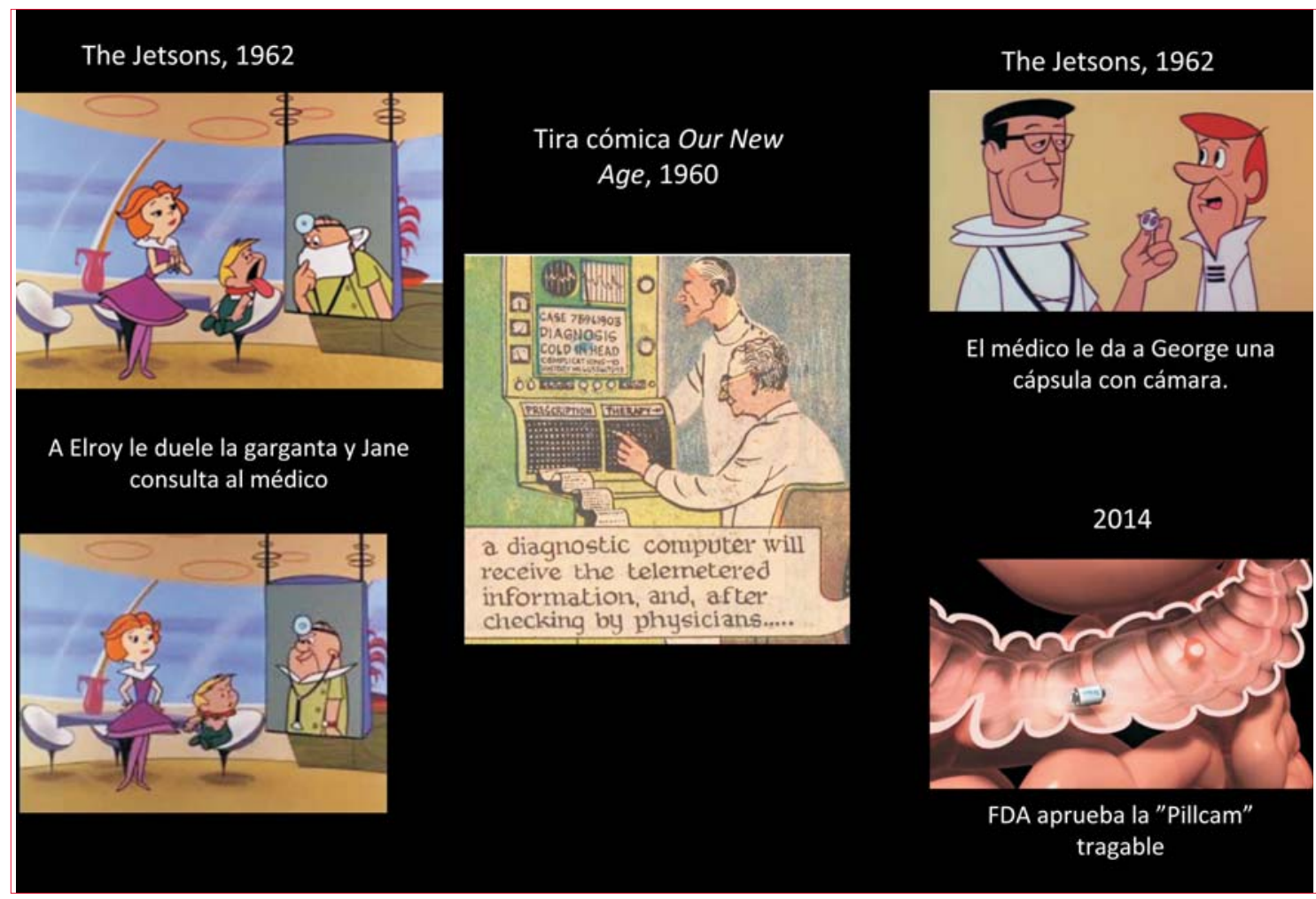

Figura 1. Fuentes:

https://www.smithsonianmag.com/history/george-jetson-gets-a-check-up-137749/

https://www.tsl.texas.gov/ld/librarydevelopments/2019/05/30/hhh-telehealth/

https://paleofuture.gizmodo.com/the-fda-just-approved-a-pillcam-the-jetsons-predicted-5-1517876149

figura 2 se intentan resumir sus alcances. Resulta útil tener presente, para evitar confusiones, las definiciones que establece la OMS de otros términos relacionados que se emplean habitualmente (tabla 1).

"Si reunimos todo el conocimiento generado desde el inicio de la civilización humana hasta 2003 tendríamos 6 exabytes de datos.

Ese volumen de información se genera hoy cada dos días". Eric Schmidt, director ejecutivo de Google (2001-2011).

El concepto de e-Salud es amplio y abarca la instrumentación y uso de sistemas digitales de registros clínicos, uso de dispositivos móviles y aplicaciones para teléfonos celulares, redes integradas, la teleconsulta y sus derivaciones y consecuencias, el uso de big data, y las prescripciones electrónicas ${ }^{(4)}$. Sus alcances sobrepasan lo puramente asistencial, incluyendo también aspectos administrativos, educativos y de investigación. Según el Dr. Topol(5), las tres áreas en las cuales se fundamenta la salud digital son la teleconsulta, las aplicaciones para celulares inteligentes y el uso de sensores y dispositivos portátiles, tres aspectos a los que nos referiremos en próximas entregas.

Más allá de las diferentes opiniones, el uso de la tecnología en la práctica médica es una realidad; la

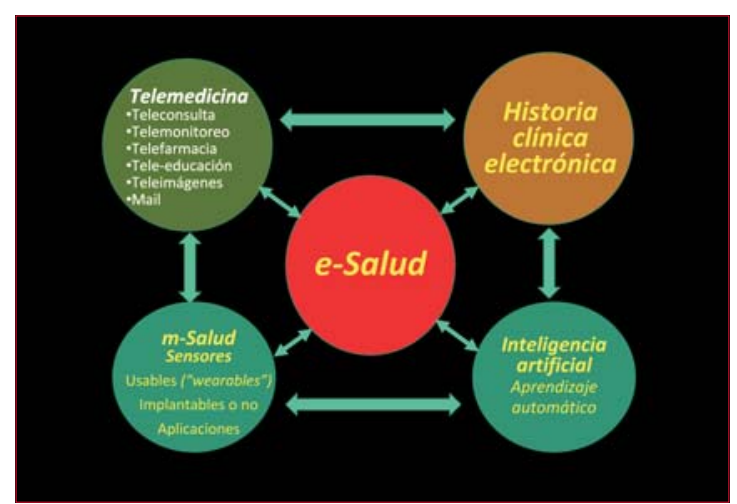

Figura 2. Representación esquemática y resumida de los conceptos que involucra el término e-Salud.

e-Salud está en fase de desarrollo explosivo acompañando la aceleración del proceso de transformación digital motivado por la crisis sanitaria. Así debería ser asumido e incorporado, idealmente a partir de una planificación e instrumentación adecuadas y de un proceso de adaptación y desarrollo progresivo y ordenado. El impulso que ha tenido en todo el mundo también ocurre en nuestro país, donde ya existen plataformas de telemedicina en funcionamiento (Doctari, por ejemplo) ${ }^{(6)}$. Nos permitió, además, tomar conciencia de que en Uruguay existen las tres condiciones indispensables para su desarrollo, acce- 


\begin{tabular}{|c|c|}
\hline e-Salud & $\begin{array}{l}\text { Uso de las tecnologías de la información y comunicación en apoyo de la salud y aspectos } \\
\text { relacionados. }\end{array}$ \\
\hline Telemedicina & $\begin{array}{l}\text { Aportar servicios de salud, donde la distancia es un factor crítico, por cualquier profesional } \\
\text { de la salud, usando las nuevas tecnologías de la comunicación para el intercambio válido de } \\
\text { información en el diagnóstico, tratamiento y prevención de enfermedades o lesiones, } \\
\text { investigación y evaluación, y educación continuada de los proveedores de salud, todo con el } \\
\text { interés de mejorar la salud de los individuos y sus comunidades }\end{array}$ \\
\hline m-Salud & Utilización de tecnologías inalámbricas móviles en salud. \\
\hline Salud digital & $\begin{array}{l}\text { Término amplio que incluye e-Salud, m-Salud, así como el uso de tecnologías avanzadas de } \\
\text { computación (big data*), genómica e inteligencia artificial. }\end{array}$ \\
\hline Telesalud & $\begin{array}{l}\text { Utilización de las telecomunicaciones y de tecnología virtual en la asistencia sanitaria } \\
\text { fuera del ámbito hospitalario o de atención tradicional. }\end{array}$ \\
\hline
\end{tabular}

so a internet de banda ancha, disponibilidad de un dispositivo para conectarse y nivel de "alfabetización digital" de la población. El desarrollo de la aplicación Coronavirus UY, que en su segunda versión también permite la comunicación de médicos con pacientes a través de videollamadas, es una clara demostración de este hecho ${ }^{(7)}$.

Las bases existen y sus ventajas son innegables; su sola enumeración exime de más análisis. Las resumimos a nuestro criterio en la tabla 2.

"La tecnología es la parte fácil, lo difícil es la gente". Hal Wolff, CEO de Healthcare Information and Management System Society (HIMSS )

\section{¿Qué está pasando en el mundo?}

La Unión Europea estableció un plan de acción 2012-2020 para el desarrollo de la e-Salud en la región con el objetivo de aumentar la eficiencia, mejorar la calidad de vida y desbloquear la innovación en el campo de la salud ${ }^{(8)}$. La Sociedad Europea de Cardiología (ESC), por su parte, también ha avanzado en la definición y alcances de los diferentes instrumentos involucrados en el término e-Salud, que se resumen en la tabla $3^{(9)}$.

Por otra parte, en Estados Unidos, en un completo informe del Ministerio de Salud al Congreso, del año 2016, se analizó en forma detallada la realidad en ese país, sus dificultades y carencias, necesidades y expectativas, resaltando la importancia de esta modalidad asistencial para el manejo de las enfermedades crónicas ${ }^{(10)}$. Se menciona que el $50 \%$ de la población de Estados Unidos padece al menos una patología crónica, a las que se dedica el 75\% del gasto en salud y son responsables del $70 \%$ de las
Tabla 2. Ventajas de la aplicación de la e-Salud.

- Universalización.

- Descentralización.

- Democratización.

- Combate la desigualdad.

- Aumenta alcance y radio de acción de la atención médica.

- Favorece una atención integral.

- Potencia la prevención.

- Elimina fronteras (distancias, de movilidad, económicas, etcétera).

- Ahorra tiempo y gastos.

- Mejora la gestión.

- Facilita análisis científicos y estadísticos.

- Acerca la asistencia a quien la requiere.

- Estimula el trabajo en red y en equipos.

- Promociona la interrelación entre instituciones y con las autoridades sanitarias.

- Permite establecer estrategias y metodologías globales.

Empodera a los pacientes.

muertes. La mayoría de estos pacientes son adultos mayores, con dificultades de movilidad y que deben visitar a su médico con frecuencia. El Colegio Americano de Cardiología (ACC) publicó en 2017 una guía estableciendo una hoja de ruta sobre la innovación de la práctica en la era de la salud digital, el big data y la llamada salud de precisión (precision 
Tabla 3. Alcances de la e-Salud de acuerdo a la Sociedad Europea de Cardiología. Resumida de ${ }^{(9)}$.

- Telemedicina y telecuidado (teleconsulta, monitoreo remoto, cuidados domiciliarios).

Sistemas de información clínica (historia electrónica, apoyo en decisiones, monitoreo de la calidad asistencial).

- Redes integradas nacionales y regionales (compartiendo referencias y prescripciones).

Registros de enfermedades

- m-Salud, incluyendo aplicaciones y su uso en particular para control de signos clínicos y educación y en salud pública para detección de pacientes o enfermedades.

Salud personalizada (p-Salud), "usables" o implantables micro o nanosensores o manejo y control de dispositivos de tratamiento (detectores de caídas, bombas de insulina, chalecos desfibriladores, por ejemplo).

- Big data.

health). Desarrolla, además, de manera extensa y detallada el compromiso del Colegio en el desarrollo e impulso a la tecnología y al respeto a los derechos de los pacientes, estableciendo pautas para aumentar el compromiso de pacientes y profesionales en este proceso(11). En forma paralela, el ACC ha desarrollado una plataforma de telemedicina para sus miembros (figura 3).

La Asociación Médica Americana (AMA) realizó una encuesta en 2016, que reiteró en 2019, sobre las motivaciones y los requerimientos de los médicos para la adopción de las herramientas digitales ${ }^{(12)}$. Sus principales conclusiones acerca de la evolución en este corto período fueron: un aumento del número de profesionales que visualizan beneficios en el uso de esas herramientas, un mayor uso de estas, sobre todo la teleconsulta y el monitoreo remoto, y un mayor conocimiento de las tecnologías emergentes, como la inteligencia artificial y otras. Recientemente, la AMA presentó un completo manual para la implementación y desarrollo de la telesalud ${ }^{(13)}$.

\section{Dificultades para la implementación de la e-Salud}

El proceso de transformación digital ha impactado nuestra vida diaria en los últimos años. Con el uso de celulares y televisores "inteligentes" y de las computadoras, desarrollamos on line muchas actividades que previamente hacíamos de forma pre-

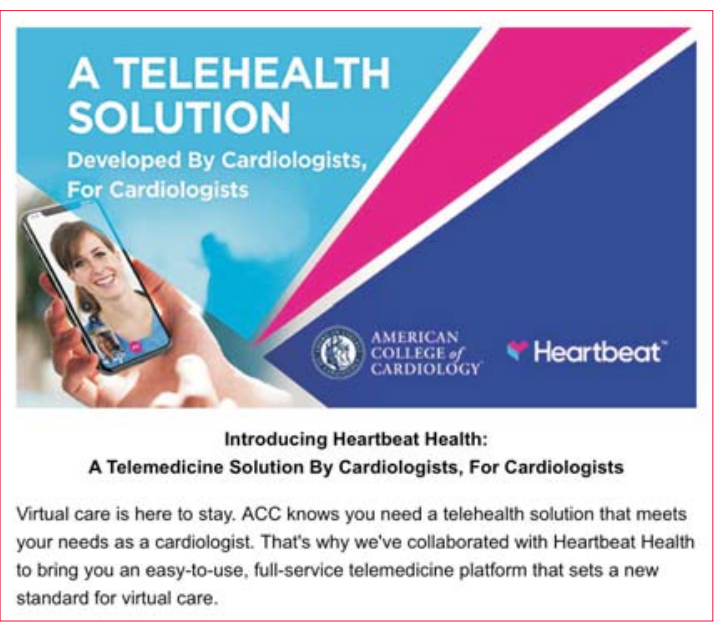

Figura 3. El Colegio Americano de Cardiología generó y ofrece a sus miembros una plataforma para Telemedicina.

sencial o con papel. A modo de ejemplo: nos comunicamos con voz e imagen con familares o amigos en el mundo, intercambiamos fotos, videos y mensajes, vemos cine, escuchamos música o radio, solicitamos comida, pedidos al supermercado, realizamos compras en el país o en el exterior, también compramos pasajes de avión, reservamos hoteles y excursiones en todas partes del mundo, leemos la prensa local e internacional, accedemos a las revistas científicas que nos interesan, estudiamos, tenemos acceso a infinitas fuentes de información, leemos o descargamos libros, etcétera. Es llamativo que con la contribución que el avance que la tecnología significó para el progreso de la medicina, fundamentalmente en las áreas de diagnóstico y tratamiento, la asistencia médica cotidiana y el uso de recursos sencillos no se haya aún generalizado para optimizar el proceso asistencial.

Existen barreras que deberían en primer término reconocerse para poder trabajar en su superación e impulsar la indispensable evolución. La más notoria es la resistencia al cambio, un espíritu conservador dominante cuando se pretende modificar algo de nuestra rutina laboral, sea esta de la calidad que sea. Frecuentemente su sola mención es percibida como una amenaza más que como un avance. Pero existen otras limitaciones, culturales, regulatorias, económicas, tecnológicas. Finalmente, quizá lo percibido como más importante, la necesidad de tener la certeza de la existencia de un marco ético y regulatorio adecuado tanto para el desarrollo de esta actividad profesional como para el respeto a los derechos de los pacientes.

En el documento antes mencionado ${ }^{(9)}$, la ESC realiza una evaluación del plan de acción 20102020, a cinco años de su inicio, identificando las principales dificultades encontradas (tabla 4), las 
Tabla 4. Limitaciones para la implementación de la e-Salud, Sociedad Europea de Cardiología. Extractada de ${ }^{(9)}$.

- Falta de conocimiento y confiabilidad en la práctica de la e-Salud.

- Falta de interrelación con colegas.

- Poca evidencia de su relación costo-beneficio.

- Ausencia de legislación en el uso de aplicaciones de salud para celulares y falta de transparencia en la seguridad de los datos recogidos.

- Respaldo legal inadecuado o fragmentado.

- Inadecuado pago por su uso.

- Diferencias regionales en el acceso a la tecnología de comunicación e información.

que motivan un llamado a los más de 80.000 cardiólogos que la integran, a apoyar el proceso. Creo que es útil tenerlas en cuenta.

"La medicina de hoy parecerá que estaba en la Edad Media comparada con lo que va a ser en el futuro". Eric Schmidt, CEO de Google (2001-2011).

\section{Breve panorama de la situación en Uruguay}

\section{Conectividad}

Nuestro país está en una situación privilegiada para instrumentar en forma óptima la e-Salud. La conectividad es muy buena y el ingreso a internet de la población ha crecido progresivamente, siguiendo una tendencia mundial. Resumiendo algunos de los últimos datos disponibles ${ }^{(14)}$ podemos apreciar que promedialmente el 88\% de los hogares tiene conexión a internet y el $71 \%$ banda ancha fija (porcentaje algo mayor en Montevideo que en el interior y también en los quintiles de mayores ingresos con respecto a los de menores ingresos) (figura 4). De cualquier manera, estas brechas se han estrechado en los últimos diez años. Además, 68\% de los hogares tiene algún dispositivo digital sin considerar los celulares. Casi $90 \%$ de las personas se conectan a internet diariamente, la mayoría desde el hogar y $98 \%$ a través del celular; $33 \%$ lo hace solo por celular (nivel educativo bajo y quintiles de menores recursos). El uso de internet se incrementó en el mundo un $70 \%$ en marzo y abril de este año. Es altamente probable que en nuestro país también se haya incrementado y quizá las cifras mencionadas subestimen la realidad actual.
Tabla 5. Telemedicina. Principios fundamentales. Ley No 18.969, abril 2, 2020.

- Universalidad

- Equidad

- Calidad del servicio

Eficiencia

- Descentralización

- Complementariedad

- Confidencialidad

\section{Legislación}

El 2 de abril de 2020 el Parlamento Nacional aprobó la ley No 19.869 titulada "Telemedicina. Normas para su implementación como prestación del servicio de salud"(15). En ella se establecen los lineamientos generales para su puesta en práctica y desarrollo. Fundamenta su implementación en una serie de principios que mostramos en la tabla 5. Se jerarquiza en esta norma, breve y concreta, que su práctica debe tener siempre el consentimiento expreso del paciente. Se respalda y refiere en su texto a leyes previas, No 18.335 (de 2008, referida a derechos y obligaciones de pacientes y usuarios de los servicios de salud), No 18.331 (de 2008 sobre protección de datos personales), y No 18.719 (ley de Presupuesto Nacional 2010-2014). Toda actividad médica en esta modalidad deberá cumplir siempre los aspectos éticos definidos claramente en la ley 19.286 del año $2014^{(15)}$.

\section{Historia clínica electrónica}

La historia clínica electrónica (HCE) es una herramienta fundamental para el funcionamiento de la e-Salud. En nuestro país, como es conocido, la Agencia de Gobierno Electrónico y Sociedad de la Información y del Conocimiento (AGESIC) lidera un proceso desde hace años para el desarrollo de una HCE nacional (HCEN) en plena evolución. El llamado Plan de Adopción 2017-2020, definido por AGESIC como "la estrategia de Salud.uy para que los efectores del Sistema Nacional Integrado de Salud se incorporen a la HCEN" aspira a que un $100 \%$ de los usuarios del sistema de salud dispongan de su HCE al final de ese período ${ }^{(16)}$.

Varias instituciones de asistencia médica también disponen de historias clínicas digitalizadas, diferentes y con distintas funcionalidades. Es interesante conocer qué uso se realiza de las diferentes prestaciones, para lo cual recurrimos al informe TIC y Salud(17) de AGESIC, del cual reproducimos 


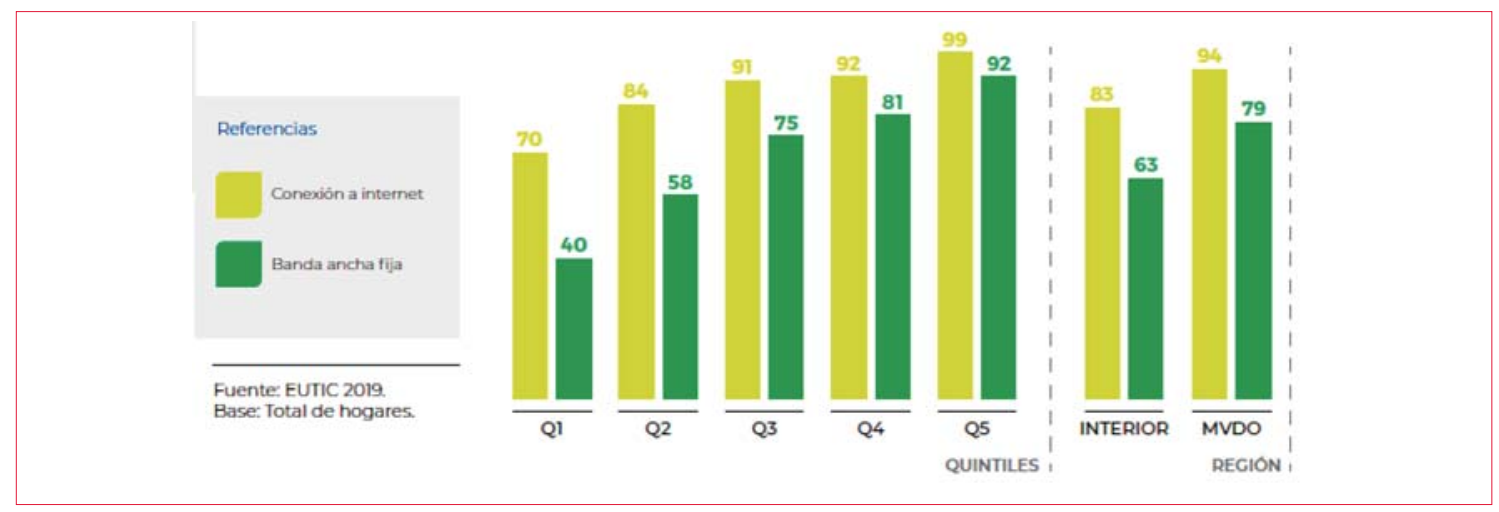

Figura 4. Conexión a internet y conexión por banda ancha fija en hogares según quintil de ingreso y región, 2019 (\%). Tomada de Encuesta de Usos de Tecnologías de la Información y Comunicación 2019 (10).

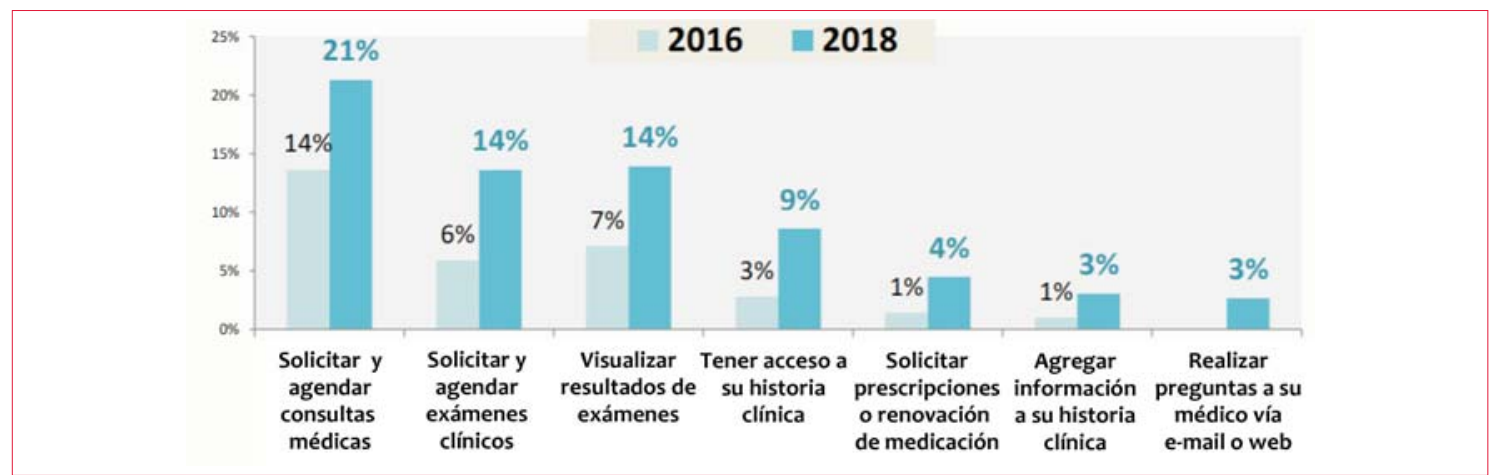

Figura 5. Uso de los servicios ofrecidos vía internet por su institución, 2016 y $2018^{(17)}$.

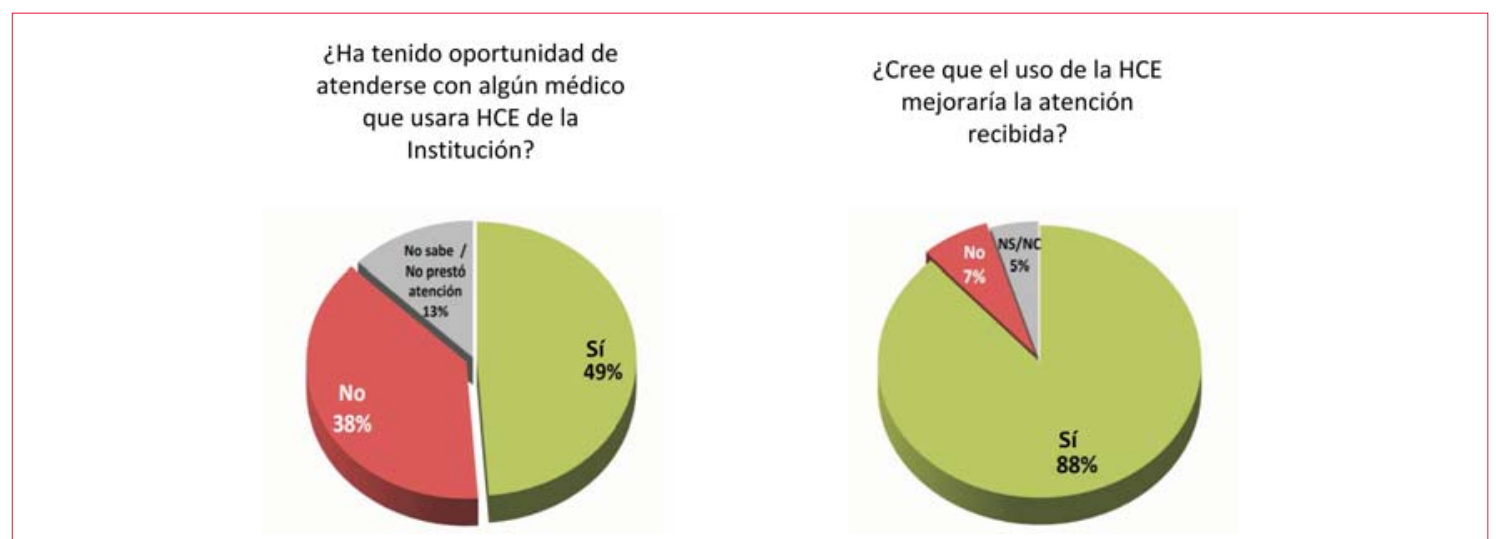

Figura 6. Uso de HCE por el médico y percepción sobre su eficacia en el usuario(17). HCE: historia clínica electrónica; NS/NC: no sabe/no contesta.

varios gráficos basados en una encuesta realizada a 800 usuarios.

La utilización de estos recursos por parte de los usuarios es todavía limitada, sobre todo en lo referido al vínculo con el médico y con su HCE (figura 5). De cualquier manera, la evolución muestra un crecimiento en los años que se comparan (2016-2018) y seguramente estos porcentajes han crecido debido al coronavirus. Aproximadamente la mitad de los médicos utilizan la HCE y la percepción del usuario al respecto es altamente positiva (figura 6). Los usuarios también perciben que el manejo de la HCE por parte del médico fue aceptable y valoran su importancia y la seguridad que transmite durante al acto asistencial (figura 7). Una mención a la situación de los Institutos de Medicina Altamente Especializada (IMAE), también considerados en este reporte. Si bien tienen una alta disponibilidad de HCE, existe muy poca vinculación con los sistemas de la institución sede u otros. 


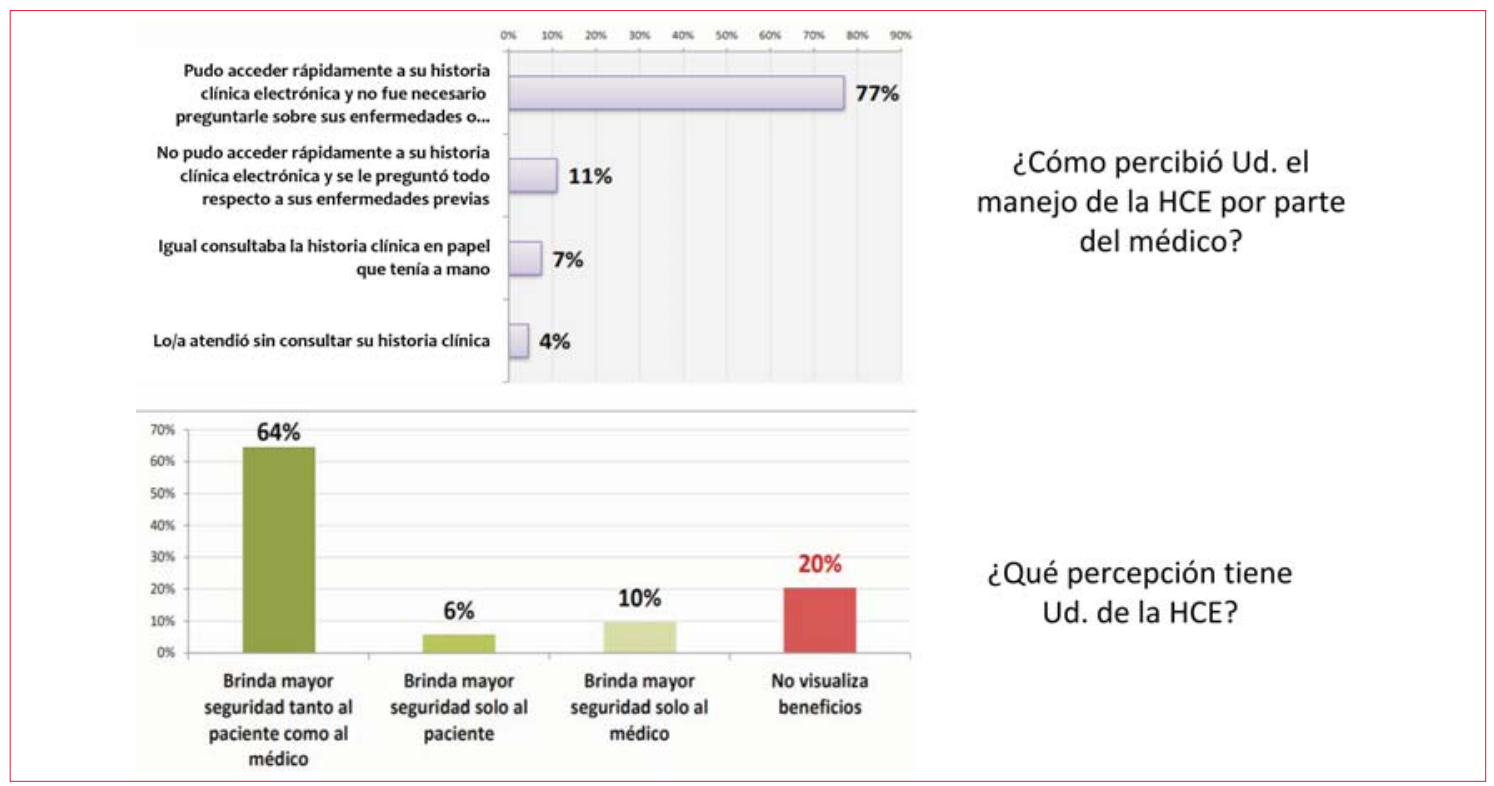

Figura 7. Cómo manejó el médico la HCE y percepción del usuario ${ }^{(17)}$. HCE: historia clínica electrónica.



Figura 8. Almacenamiento de los datos por las instituciones ${ }^{(17)}$. NS/NC: no sabe/no contesta.

La confidencialidad y la seguridad de los datos son factores clave en este proceso, sobre los cuales debe trabajarse permanentemente con especialistas en cada institución, evaluando y corrigiendo en forma permanente. Un aspecto vinculado es el relativo al almacenamiento de la información y los registros de los pacientes por parte de las instituciones (figura 8). Solamente el $13 \%$ de las instituciones tiene un registro exclusivamente digital, y $9 \%$ aún archiva solo en papel. El porcentaje mayor comparte entre papel y digital en distintas proporciones. $\mathrm{Si}$ bien la situación todavía dista de ser ideal, se está en un proceso de avance notorio si se considera el período 2014-2018.

\section{¿Qué opinan los profesionales de la salud sobre la historia clínica electrónica?}

Aun en países donde la HCE es de uso generalizado desde hace mucho tiempo, siguen existiendo controversias y las opiniones son diversas. La Universidad de Stanford, Estados Unidos, realizó en el año 2018 una encuesta sobre una muestra de médicos de atención primaria que tuvieran más de un mes 
Comparte su contraseña con otros colegas o personal asistencial.

Conecta su equipo personal (notebook, Tablet, etc.) a la red de la institución.

Trabaja con información clínica y/o mantiene copias en sus equipos personales.

Tiene permitido instalar programas en su equipo de trabajo provisto por la institución.

Comparte información clínica para interconsulta $u$ otros motivos por medios no institucionales.

En su institución, utiliza usuarios genéricos para acceder a los sistemas o aplicativos.

Desde el sistema puede acceder a $\mathrm{HC}$ de un paciente sin tener cita u orden de servicio generada.

\section{ENunca 1 Algunas veces}
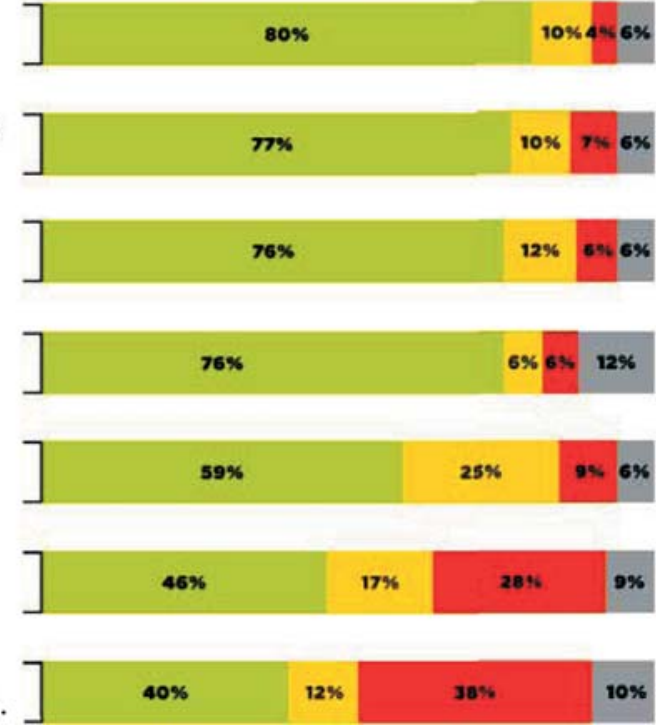

Figura 9. Datos importantes sobre el uso de los sistemas informáticos, relativos a la confiabilidad y seguridad de los mis$\operatorname{mos}^{(17)}$. NS/NC: no sabe/no contesta; HC: historia clínica.

de experiencia en el uso de la HCE, seleccionados a partir del padrón de la $\mathrm{AMA}^{(18)}$. El resultado en términos generales fue muy crítico de la realidad, evidenció una serie de problemas y la necesidad de mejora. Dos tercios de los entrevistados opinaron que si bien ha contribuido a mejorar la calidad asistencial y están satisfechos, persisten algunos problemas. El $62 \%$ del tiempo dedicado a cada paciente es utilizado con la HCE y una mayoría considera que ha ocasionado un aumento de su trabajo. Solo el $8 \%$ piensa que su objetivo primario sea clínico, mientras el $44 \%$ percibe que su finalidad principal es el almacenamiento de datos.

Teniendo presente que luego de la experiencia de la pandemia, las opiniones, tanto en nuestro país como en el mundo podrían haber variado, mencionaremos los datos que hemos podido relevar de Uruguay al respecto. La información también surge del documento TIC y Salud 2018 presentado por la AGESIC en diciembre de 2019(17). Se tomó una muestra aleatorizada estratificada de manera no proporcional que incluyó a 200 médicos, 200 licenciados en enfermería y 200 auxiliares de enfermería. Globalmente el $80 \%$ se conecta diariamente con la HCE durante la asistencia de un paciente, $75 \%$ trabaja en instituciones que disponen de HCE y el $64 \%$ la utiliza siempre. Cuatro de cada diez profesionales consultan diariamente algún dato (resultados de laboratorio, diagnósticos, motivos de consulta o anotaciones clínicas), siendo la frecuencia mayor en los profesionales del interior con respecto a la capital. Aproximadamente la mitad ha recibido capacitación, siendo algo mayor el porcentaje en licen- ciados en enfermería. Como se aprecia en la figura 8 , en lo relativo a la seguridad y confidencialidad de los datos, si bien en algunos rubros la conducta del personal de la salud es aceptable, en otros no es así y debería trabajarse en su profundización en todos.

"Cuando el porcentaje de personas mayores que manejen un 'smartphone' o una 'tablet' se eleve (lo hará de manera clara en los proximos años), debemos estar preparados para poder atender mejor a los pacientes, de una forma más cómoda, más eficaz y sobre todo más sostenible". Dr. Rafael Vidal Pérez, cardiólogo español.

\section{Conclusiones}

Compartimos las conclusiones del documento de Consenso 2020 recientemente publicado por la Sociedad del Ritmo Cardíaco (HRS), la Sociedad del Ritmo Cardíaco de la región Asia-Pacífico (APHRS) y de Latinoamérica (LAHRS), el ACC y la Asociación Americana del Corazón (AHA)(19).

"La crisis precipitada por la pandemia ha catalizado la adopción del manejo remoto del paciente en muchas especialidades y para los especialistas en arritmias en particular. Esta práctica llegó para quedarse. Es una oportunidad para integrar y aumentar el uso de los servicios remotos en la práctica médica en el mundo"(13).

\section{Walter Reyes Caorsi,}

https://orcid.org/ 0000-0002-4670-5765

Este artículo fue aceptado para su publicación por: Editora jefa anterior Dra. María del Pilar Aguilar Passano. 


\section{Bibliografía}

1. World Health Organization. Telemedicine: Opportunities and developments in member states: Report on the second global survey on eHealth [Internet]. Ginebra: WHO; 2009 [consulta 14 Set 2020]. Disponible en: http://www.who.int/goe/publications/goe_telemedicine_2010.pdf

2. Willem Einthoven: as part of our ongoing series of Novel Prices winners that have contributed to cardiovascular medicine, Mark Nicholls focuses on the work of Willem Einthoven who was awarded the 1924 Nobel Rrize in physiology or medicine "for his discovery of the mechanism of the electrocardiogram". Eur Heart J. 2019;40(37):3075-8. doi:10. 1093/eurheartj/ehz663

3. World Health Organization. WHO guideline: recommendations on digital interventions for health system strengthening: evidence and recommendations [Internet]. Geneva: WHO; 2019 [consulta 14 Set 2020]. Disponible en: https://apps.who.int/iris/ handle/10665/311980

4. Singhal A, Cowie M. What is e-Health? e-Journal of Cardiology Practice. 2020 Jun [consulta 14 Set 2020];18(24). Disponible en: https://www.escardio. org/Journals/E-Journal-of-Cardiology-Practice/Volume-18/what-is-e-health

5. Topol E. The Topol Review. Preparing the healthcare workforce to deliver the digital future [Internet] Leeds: Health Education England; 2019 [consulta 14 Set 2020]. Disponible en: file://C:/Users/medina/ AppData/Local/Temp/HEE-Topol-Review-2019.pdf

6. Malek M. Telemedicina: creció con la pandemia y llegó para quedarse [Internet]. Montevideo: Diario El País. Montevideo; 2020 [consulta 14 Set 2020]. Disponible en: https://www.elpais.com.uy/vida-actual/ telemedicina-crecio-pandemia-llego-quedarse.html

7. Telemedicina: salud y tecnología al servicio de la ciudadanía [Internet]. Montevideo: Presidencia de la República; 2020 [consulta 14 Set 2020]. Disponible en https://www.gub.uy/agencia-gobierno-electronico-sociedad-informacion-conocimiento/comunicacion/noticias/telemedicina-salud-tecnologia-servicio-ciudadania

8. European Commission. Communication from the Commission of the European Parliament, the Council, the European Economic and Social Committee, and the Committee of the Regions eHealth Action Plan 2012-2020 - innovative healthcare for the 21st Century. Brussels: European Comission; 2012 [consulta 14 Set 2020]. Disponible en: http://ec.europa.eu/information society/newsroom/cf/dae/document.cfm?doc_id $=4188$

9. Cowie MR, Bax J, Bruining N, Cleland JGF, Koehler F, Malik M, et al. e-Health: a position statement of the European Society of Cardiology. Eur Heart J. 2016; 37(1): 63-6. doi:10.1093/eurheartj/ ehv416
10. Report to Congress: e-health and telemedicine [Internet]. Washington: US Departament of Health and Human Services ; 2016 [consulta 26 Jul 2020]. Disponible en: https://aspe.hhs.gov/pdf-report/report-congresse-health-and-telemedicine.

11. Bhavnani SP. Parakh K, Atreja A, Druz R, Graham GN, Hayek SS, et al. 2017 road map for innovation- ACC health policy statement, on healthcare transformation in the era of digital health, big data and precision health. A report of the American College of Cardiology task force on health policy statements and systems of care. J Am Coll Cardiol. 2017;70(21): 2696-718. doi: 10.1016/j.jacc.2017.10. 018

12. AMA digital health research. Physicians' motivations and requirements for adopting digital health adoption and attitudinal shifts from 2016 to 2019 [Intenet]. Chicago: American Medical Association; 2020 [consulta 16 Set 2020]. Disponible en: https://www.ama-assn.org/system/files/2020-02/ ama-digital-health-study.pdf

13. Telehealth Implementation Playbook [Intenet]. Chicago: American Medical Association; 2020 [consulta 16 Set 2020]. Disponible en: https://www. ama-assn.org/system/files/2020-04/ama-telehealthplaybook.pdf

14. Estadísticas. Estudio sobre conocimientos, actitudes y prácticas de ciudadanía digital 2020 [Internet]. Montevideo: Presidencia de la República; 2020 [consulta 14 Set 2020]. Disponible: https://www.gub. uy/agencia-gobierno-electronico-sociedad-informacion-conocimiento/datos-y-estadisticas/estadisticas

15. Telemedicina, implementación y desarrollo. Régimen. Ley № 19869. (Abr 2, 2020). Disponible en: https://parlamento.gub.uy/documentosyleyes/fichaasunto/144552

16. Plan de adopción HCEN iQué es? [Internet]. Montevideo: Agesic;2020 [consulta 16 Set 2020]. Disponible en: https://centrodeconocimiento.agesic.gub.uy/web/ salud.uy $/ \% \mathrm{C} 2 \% \mathrm{BFqu} \% \mathrm{C} 3 \% \mathrm{~A} 9-\mathrm{es}-$

17. Medición de TICy salud [Internet]. Montevideo: Agesic;2020 [consulta 16 Set 2020]. Disponible en: https://www.gub.uy/agencia-gobierno-electronicosociedad-informacion-conocimiento/node/342

18. Poll H. How doctors feel about electronic health records: National Physician Poll [Internet]. Stanford: Stanford Medicine; 2018 [consulta 24 Jun 2020]. Disponible en: http://med.stanford.edu/content/dam/ sm/ehr/documents/EHR-Poll-Presentation.pdf

19. Varma N, Marrouche NF, Aguinaga L, Albert CM, Arbelo E, Choi J, et al. HRS/APHRS/LAHRS/ ACC/AHA worldwide practice update for telehealth and arrhythmia monitoring during and after a pandemic. J Am Coll Cardiol. 2020; S0735-1097(20) 35623-0. doi: 10.1016/j.jacc.2020.06.019 